\title{
F. Application of Neurophygiology to the Neurosurgical Field
}

\section{F-1. Automated EEG and EVR Processings for Evaluation of Disturbed Consciousness with Visual Displays}

\author{
Masayoshi Kowada, M. D. \\ Division of Surgical Neurology, Research Institute of Brain \\ and Blood Vessels, Akita
}

Long-term serial electroencephalograms and visual evoked responses have been processed automatically by a general purpose digital computer (JEC-7) in order to evaluate the consciousness level of patients in coma. Processed data have been displayed on the CRT or with the digital plotter for the visual convenience.

After a basic pattern of an electroencephalogram has been recognized by a combined cross-over and alternative method, an histogram of a curtain duration has been converted into "numerical representation" and has been plotted in a difined three dimentional space as a flying spot. "Clock" and "colloseum" display have been made for the convenience to observe an outline of a long-term electroencephalographic changes.

Sixty-four serial visual evoked responses have been superimposed obliquely on the CRT as "bird-eye" display and each deflexion has been automatically recognized. Twenty-four visual evoked responses recorded on the radial line at the occipital region have been processed and projected in a difined space. Above-described methods of automated processing of clinical electroencephalograms or visual evoked responses have been reported in detail especially from the clinical point of the visual display.

\section{F-2. Neurophysiological Observations in Two Cases Showing Prolonged Goma}

\author{
Motohide Ogashiwa and Yoshikazu Sarto \\ Division of Neurosurgery, Institute of Neurological Sciences, \\ Tottori University School of Medicine
}

An analisis of the behavioral functions by several physiological techniques were performed in two cases showing prolonged coma. The prolonged coma of the cerebral type due to head injury and of the brain stem type due to clivus meningioma was observed and the differences of neurophysiological findings were discussed. 